Related content

- Nonlinear quantum optics mediated by
$\frac{\text { Rydberg interactions }}{\text { O Firstenberg, C S Adams and S }}$
Hofferberth
- $\frac{\text { Rydberg-induced optical nonlinearities }}{\text { from a cold atomic ensemble trapped }}$
$\frac{\text { inside a cavity }}{\text { R Boddeda, I Usmani, E Bimbard et al. }}$
- Quantum statistics of light transmitted
$\frac{\text { through an intracavity Rydberg medium }}{\text { A Grankin, E Brion, E Bimbard et al. }}$

LETTER

\section{Conditional phase-shift enhancement through dynamical Rydberg blockade}

To cite this article: Jin-Hui Wu et al 2017 EPL 12054002

View the article online for updates and enhancements. 


\title{
Conditional phase-shift enhancement through dynamical Rydberg blockade
}

\author{
Jin-Hui $\mathrm{Wu}^{1}$, M. Artoni ${ }^{2,3}$, F. Cataliotti ${ }^{2,4}$ and G. C. La Rocca ${ }^{5}$ \\ 1 Center for Quantum Sciences and School of Physics, Northeast Normal University - Changchun 130024, China \\ 2 European Laboratory for Nonlinear Spectroscopy (LENS) - 50019 Florence, Italy \\ 3 Department of Engineering and Information Technology and Istituto Nazionale di Ottica (INO-CNR), \\ Brescia University - 25133 Brescia, Italy \\ 4 Department of Physics and Astronomy, University of Florence - 50019 Florence, Italy \\ 5 Scuola Normale Superiore and CNISM - 56126 Pisa, Italy
}

received 31 August 2017; accepted in final form 26 January 2018

published online 22 February 2018
PACS 42.50. Gy - Effects of atomic coherence on propagation, absorption, and amplification of light; electromagnetically induced transparency and absorption
PACS 32.80.Ee - Rydberg states
PACS $42.65 .-\mathrm{k}$ - Nonlinear optics

\begin{abstract}
Large cross-phase shifts per photon can be attained through an all-optical polarization control of dipole blockade in Rydberg atoms. A pair of weak circularly polarized signal and control light pulses experience a giant nonlinear cross-interaction through the conditional excitation of a Rydberg state. Conditional cross-phase modulations on the order of $\pi$-radians may be attained under specific symmetric EIT quasi-resonant driving conditions at large degrees of transparency. We also suggest the possibility of extending our scheme to work at very low intensities and within a few-blockade-radii regions.

Copyright (C) EPLA, 2018
\end{abstract}

Introduction. - Photons are ideal carriers of information because they can easily be transmitted over long distances and loosely couple to the environment, yet their use is often largely hampered by the absence of significant photon-photon interactions especially when crossphase nonlinearities are needed [1,2]. Effective interactions between photons must be then mediated by a suitable medium to reach useful cross-phase shifts. For potential applications in advanced optical information processing [3] one is required to deal with conditional nonlinear interactions that are enabled when a "control" light pulse imprints a phase shift onto another "signal" light pulse [4]. Promising strategies consist in coupling an optical cavity to single atoms [5,6], atomic ensembles [7], and artificial atoms [8]. Alternative approaches comprise light-atoms interfaces [9] driven into a regime of electromagnetically induced transparency (EIT) [10]. These implementations are challenging though the observed cross-nonlinearities yield conditional phase shifts far less than the desired value of $\pi[11,12]$. Cross-phase modulations in the range $1-10$ micro-radians per photon have been observed in slow-light cold atoms [13] while slightly larger phase shifts have been achieved through specific post-selection procedures [14].
Rydberg atoms, on the other hand, have attracted extensive attention owing to the presence of strong dipoledipole interactions $[15,16]$. These manifest themselves directly through a dipole blockade effect $[15,17]$ preventing the simultaneous excitations of two or more atoms within a Rydberg superatom (SA) [18]. Such a mechanism has been exploited to create fairly robust lightatoms interfaces $[9,19,20]$ where the combination with EIT makes Rydberg media appealing to foster significant cooperative optical nonlinearities $[7,16,21,22]$. Recently a single-photon $\pi$ phase shift [23] has been measured in such Rydberg-EIT media through a pulse storage-retrieval technique entailing large absorptive $\operatorname{losses}^{1}$, as well as in high-finesse optical resonators for atoms [24,25].

Here we show that large conditional cross-phase shifts per signal photon can be attained over lengths of a few Rydberg SAs with weak and freely propagating signal and control pulses. Conditional $\pi$ phase modulations are found to occur over a wide parameter range and at relatively small optical depths. This hinges on polarization-selective

\footnotetext{
${ }^{1}$ The $\pi$ phase-shifted post-selected signal photon in [23] is observed upon the detection of a control photon exhibiting more than $90 \%$ absorption.
} 
nonlinearities occurring in cold Rydberg atoms under a specific EIT-symmetric driving regime [26] and is at variance with cross-Kerr like [27], resonant absorbing [28], transversely separated [29], and site addressable [30] Rydberg nonlinear mechanisms. In the EIT-symmetric driving approach pursued here the pulsed regime is further shown to benefit from intrinsic group-velocity matching and high spatial-temporal coherence of the signal and control beams. We finally discuss the feasibility of extending our scheme to work with low photon-numbers pulses subject to small losses and distortions, a critical step toward the implementation of a deterministic low-intensity optical gate $[31,32]$.

Phase shift. - We use cold Rydberg ${ }^{87} \mathrm{Rb}$ atoms driven by a pair of strong continuous-wave (CW) coupling and dressing fields into the level configurations shown in fig. 1 to manage the phases of another pair of weak signal and control pulsed fields. Our scheme relies on three key features. First, the uppermost Rydberg level $|r\rangle$ coupled to the intermediate excited level $|e\rangle$ by the strong CW dressing field $\Omega_{D}$ provides the large nonlinear mechanism. Second, the unpopulated ground-state level $|a\rangle$ coupled to the intermediate excited level $|e\rangle$ by the strong CW coupling field $\Omega_{C}$ ensures that both the signal $\left(\Omega_{s}\right)$ and control $\left(\Omega_{c}\right)$ pulses always propagate in the EIT regime. Third, the equally populated ground-state levels $|g\rangle$ and $|m\rangle$ allow for the symmetric response [33] experienced by the signal and control pulses (their role could actually be exchanged); this, in particular, turns out to be crucial for their group-velocity matching.

Upon impinging on a sample of length $L$, a monochromatic signal beam of wavevector $k_{s}=\omega_{s} / c$ will acquire the nonlinear phase shift $\phi_{s}=\phi_{o} \operatorname{Re}\left[\chi_{s}\right]$, where $\phi_{o}=k_{s} L / 2$ is half the vacuum phase shift, and will experience absorption characterized by half the nonlinear optical depth $\kappa_{s}=\phi_{0} \operatorname{Im}\left[\chi_{s}\right]\left(\left|\chi_{s}\right| \ll 1\right)$. The signal susceptibility $\chi_{s}$ is found to depend critically on whether the Rydberg transition is allowed or blocked. The latter case occurs when one atom is excited to the Rydberg level and strong dipole-dipole interactions shift the level $|r\rangle$ of other atoms within a SA far-off-resonance from the dressing field $\Omega_{D}$ (dipole blockade) [15]. When the control beam is present with the $\sigma_{c}^{-}$polarization (see fig. 1(a)), atoms inside a SA are driven into a " $\Phi$ " or a " $\Pi$ " configuration respectively in the limit of small $\left(P^{a} \rightarrow 0\right)$ or large $\left(P^{a} \rightarrow 1\right)$ SA Rydberg excitations. Similarly, when the control beam is absent with the $\sigma_{c}^{+}$polarization (see fig. $1(\mathrm{~b})$ ), atoms inside a SA are driven into a " $X$ " or a " $\Lambda$ " configuration in the limit of small $\left(P^{b} \rightarrow 0\right)$ or large $\left(P^{b} \rightarrow 1\right)$ SA Rydberg excitations. The simpler " $m$ " and " $\Lambda$ " configurations occur because of dipole-blockade and most importantly, the SA Rydberg populations themselves strongly depend on whether the control beam is present $\left(P^{a}\right)$ or absent $\left(P^{b}\right)$, which triggers a very large cross-nonlinearity.

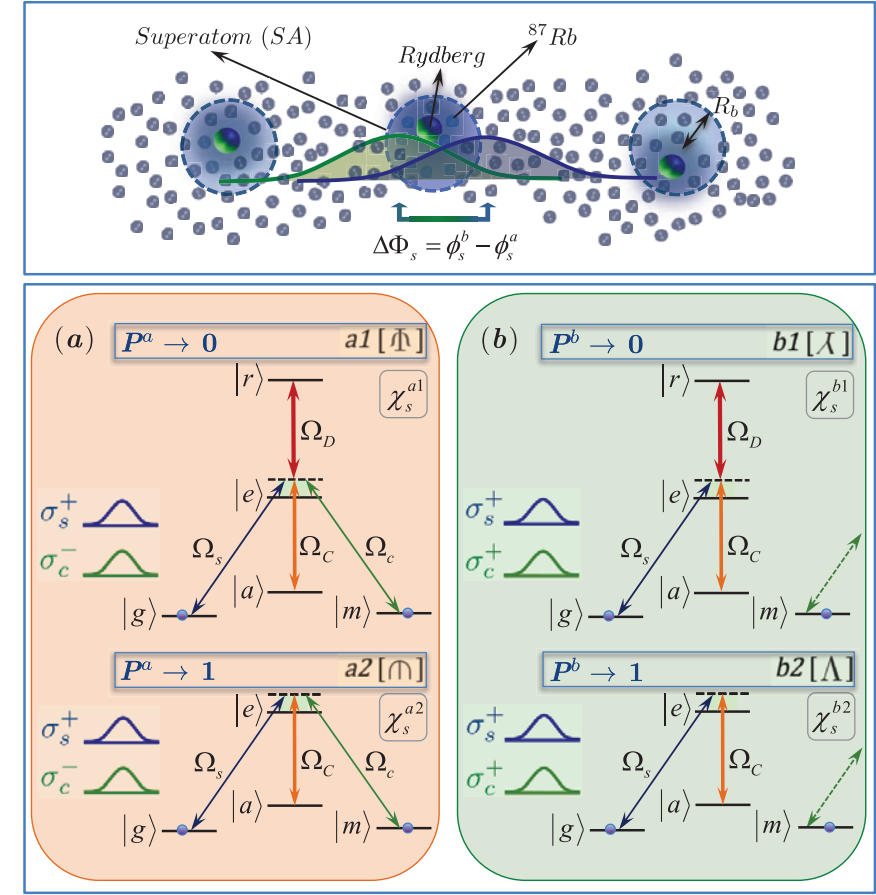

Fig. 1: (Color online) Top: conditional cross-phase shift. Different phase shifts $\phi_{s}^{a}$ and $\phi_{s}^{b}$ are imprinted upon a signal beam propagating across a SA, depending on the presence or absence of a control beam. The conditional cross-phase shift $\Delta \Phi_{s}$ may equal $\pi$ for tens of SAs under a suitable symmetric-EIT configuration (see text). Bottom: cooperative signal susceptibilities. Level configurations contributing to different cooperative signal susceptibilities, depending on the presence (a1-a2) or absence (b1-b2) of the control beam, being either choice conditional to its circular polarization. Each cooperative signal susceptibility is further determined by the SA population of the Rydberg state $|r\rangle$, whose two opposite limits $P^{a, b} \rightarrow 0$ and $P^{a, b} \rightarrow 1$ result in different level configurations and thus different individual signal susceptibilities $\chi_{s}^{a 1, b 1}$ and $\chi_{s}^{a 2, b 2}$. The levels $\{|g\rangle,|a\rangle,|m\rangle,|e\rangle$, and $|r\rangle\}$ represent the ${ }^{87} \mathrm{Rb}$ manifold $\left\{\left|5^{2} S_{1 / 2}, F=1, m=-1\right\rangle, \quad\left|5^{2} S_{1 / 2}, F=2, m=0\right\rangle\right.$, $\left|5^{2} S_{1 / 2}, F=1, m=+1\right\rangle,\left|5^{2} P_{1 / 2}, F=1, m=0\right\rangle$, and $\left.|90 s\rangle\right\}$ whose detunings from the dressing, coupling, signal, and control fields are $\delta_{D}=\omega_{D}-\omega_{r e}, \delta_{C}=\omega_{C}-\omega_{e a}, \delta_{s}=\omega_{s}-\omega_{e g}$, and $\delta_{c}=\omega_{c}-\omega_{e m}$ in order with $\Omega_{D, C, s, c}$ denoting the corresponding Rabi frequencies.

Such polarization-selective Rydberg nonlinearities can, in fact, be exploited to bring about large conditional changes in the signal phase over a wide range of the coupling and dressing Rabi frequencies $\left(\Omega_{C, D}\right)$ and detunings $\left(\delta_{C, D}\right)$. In what follows, we choose to work with equal signal and control detunings $\delta_{s}=\delta_{c} \equiv \delta$, Rabi frequencies $\Omega_{s}=\Omega_{c}$, and ground levels $\{|g\rangle,|m\rangle\}$ populations, i.e., with a symmetric-EIT driving configuration (see the Supplementary Material Supplementarymaterial.pdf (SM) for details). We further adopt a universal relation for the dependence of the signal susceptibility $\chi_{s}$ on the SA Rydberg population $P^{a, b}$ akin to the one introduced in [17]. Then the signal phase shift in the presence $\left(\phi_{s}^{a}\right)$ or 
absence $\left(\phi_{s}^{b}\right)$ of the control beam can be written $\mathrm{as}^{2}$

$$
\begin{aligned}
& \phi_{s}^{a}=\phi_{0}\{\underbrace{P^{a} \operatorname{Re}\left[\chi_{s}^{a 2}\right]+\left(1-P^{a}\right) \operatorname{Re}\left[\chi_{s}^{a 1}\right]}_{\operatorname{Re}\left[\chi_{s}^{a}\right]}\}, \quad\{a 1 \rightleftharpoons a 2\}, \\
& \phi_{s}^{b}=\phi_{0}\{\underbrace{P^{b} \operatorname{Re}\left[\chi_{s}^{b 2}\right]+\left(1-P^{b}\right) \operatorname{Re}\left[\chi_{s}^{b 1}\right]}_{\operatorname{Re}\left[\chi_{s}^{b}\right]}\}, \quad\{b 1 \rightleftharpoons b 2\},
\end{aligned}
$$

Such relations are found to agree with rate equation models of multilevel Rydberg atoms exhibiting strong dipole-dipole interactions, based both on many-body simulations [17] and on semi-analytical one-body approaches [34]. In fact, they have been used to explain the observation of nonlinear dispersive effects in cold Rydberg atoms $[7,35]$. The phase shift $\phi_{s}^{a}$ depends on the susceptibility $\chi_{s}^{a}$ which we term here cooperative as it is determined by the interplay of the two individual susceptibilities $\chi_{s}^{a 1}$ and $\chi_{s}^{a 2}$ (see SM for details). The susceptibility $\chi_{s}^{a}$ strongly depends on the SA Rydberg population $P^{a}$ provided $\chi_{s}^{a 1}$ is significantly different from $\chi_{s}^{a 2}$. The same holds for $\phi_{s}^{b}$.

The population $P^{a}$ specifically represents the averaged Rydberg excitation probability (see SM for details), i.e., the Rydberg excited fraction for a single SA containing $n_{S A}$ atoms [34]. In the presence of the control beam, for weak dressings the Rydberg population may decrease to $P^{a} \rightarrow 0$ so that the signal experiences the five-level ( $\left.\Phi\right)$ dispersive shift $\phi_{o} \operatorname{Re}\left[\chi_{s}^{a 1}\right]$; conversely for intense dressings the Rydberg population may increase to $P^{a} \rightarrow 1$ so that the signal experiences the four-level $(\Pi)$ dispersive shift $\phi_{o} \operatorname{Re}\left[\chi_{s}^{a 2}\right]$. The latter is based on the fact that an atom excited to the Rydberg level can detune from resonance all neighboring atoms inside a blockade sphere of radius $R_{b}[34]^{3,4}$. Analogous considerations hold for the population $P^{b}$ and for the shift $\phi_{s}^{b}$ in eq. (2) which will reduce in one case to $\phi_{o} \operatorname{Re}\left[\chi_{s}^{b 1}\right](X)$ and in the other case to $\phi_{o} \operatorname{Re}\left[\chi_{s}^{b 2}\right](\Lambda)$. It is further worth noting that for the specific symmetric-EIT driving configuration considered here, the signal exhibits almost identical responses for the $\Lambda$ (b2) and the $\cap(a 2)$ configurations as well as for the $\Lambda(b 1)$ and the $\Phi(a 1)$ configurations $^{5}$. The conditional

\footnotetext{
${ }^{2}$ Similarly, replacing $\operatorname{Re}[] \rightarrow \operatorname{Im}[]$ in eqs. (1) and (2) yields the absorption coefficients $\kappa_{s}^{b}$ and $\kappa_{s}^{a}$.

${ }^{3} R_{b}=\sqrt[6]{C_{6}\left|\delta_{D}\right| /\left(\left|\Omega_{C}\right|^{2}+\left|\Omega_{D}\right|^{2}\right)}$ is defined here by considering $V\left(R_{b}\right)=\hbar \gamma_{E I T}$ with $V(R)=\hbar C_{6} / R^{6}$ being the van der Waals potential at distance $R$ and $\gamma_{E I T}=\left(\left|\Omega_{C}\right|^{2}+\left|\Omega_{D}\right|^{2}\right) /\left|\delta_{D}\right|$ being the EIT linewidth associated with Rydberg excitation in the case of $\left|\delta_{D}\right| \gg \gamma_{g e}[21]$. We have also considered in calculations that SA interactions will result in a reduction of the blockade radius [34], e.g., from $R_{b} \simeq 13.97 \mu \mathrm{m}\left(n_{s a}=27350\right)$ to $R_{b} \simeq 13.27 \mu \mathrm{m}\left(n_{s a}=23250\right)$ for the optimal parameters.

${ }^{4}$ The concept of an excitation blockade sphere [7,17], adopted here to get an intuitive picture of effects of the dipole-dipole interaction on the signal (control) quantum coherence, enables us to include Rydberg blockade effects in the cooperative susceptibilities $\chi_{s}^{a, b}$ in eqs. (1) and (2).

${ }^{5}$ This has also been confirmed through numerical computations for the realistic Rydberg blockade sample we examine here (see SM for details).
}
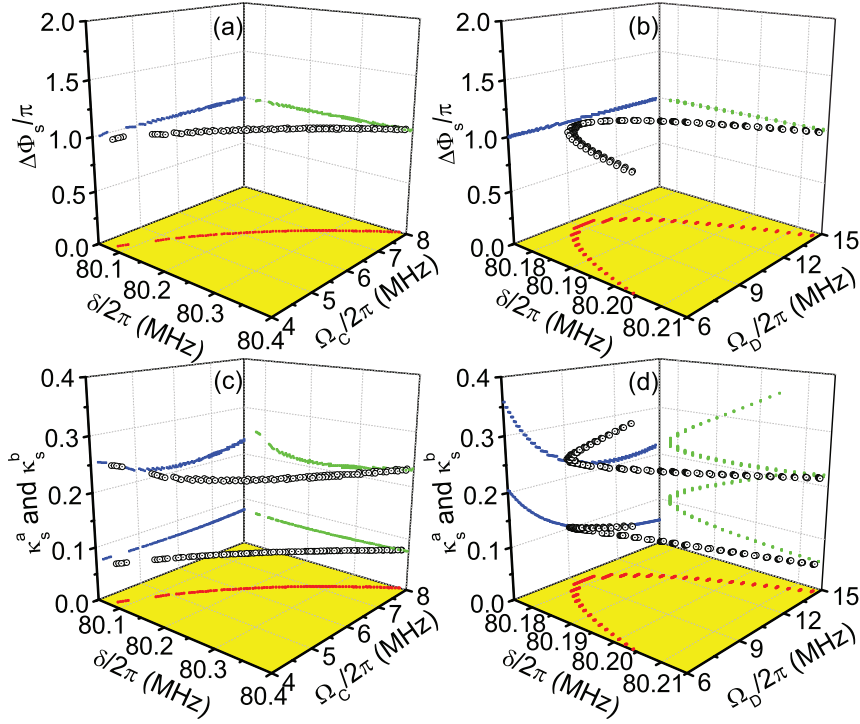

Fig. 2: (Color online) Cross-phase shifts ((a), (b)) and half-optical depths ((c), (d)) for CW signal and control fields vs. $\delta$ and $\Omega_{C}$ with $\Omega_{D}=2 \pi \times 12.0 \mathrm{MHz}((\mathrm{a}),(\mathrm{c})) ; \delta$ and $\Omega_{D}$ with $\Omega_{C}=2 \pi \times 6.0 \mathrm{MHz}((\mathrm{b}),(\mathrm{d}))$. Black points in ((a), (b)) show the parameter regions where $\Delta \Phi_{s}=\pi$ along with the (blue-red-green) coordinate-planes projections. Black points in ((c), (d)) show the half-optical depths corresponding to $\Delta \Phi_{s}=\pi$ along with the (blue-red-green) coordinateplanes projections. The upper (lower) set of black points in $((c),(d))$ are obtained in the absence (presence) of the control beam. The sample of cold ${ }^{87} \mathrm{Rb}$ atoms has a length $L=1.0 \mathrm{~mm}$, a density $N_{0}=4.8 \times 10^{12} \mathrm{~cm}^{-3}$, dipole moments $d_{e g}=d_{e m}=1.5 \times 10^{-29} \mathrm{~cm}$, and homogeneous dephasings: $\gamma_{g e, m e, a e, r e}=2 \pi \times 3.0 \mathrm{MHz}, \gamma_{g r, m r, a r}=2 \pi \times 10 \mathrm{kHz}$, and $\gamma_{g a, g m, m a}=2 \pi \times 2.0 \mathrm{kHz}$. The CW coupling and dressing fields have detunings $\delta_{C}=-\delta_{D}=2 \pi \times 80.0 \mathrm{MHz}$.

phase shift, i.e., the difference between the signal phase shift when the control is present $(a 1 \rightleftharpoons a 2)$ and the signal phase shift when the control is absent $(b 1 \rightleftharpoons b 2)$, then becomes

$$
\Delta \Phi_{s}=\left(\phi_{s}^{b}-\phi_{s}^{a}\right) \simeq \phi_{0} \operatorname{Re}\left[\chi_{s}^{b 2}-\chi_{s}^{b 1}\right] \times\left(P^{b}-P^{a}\right),
$$

where the challenge is to achieve $\left|\Delta \Phi_{s}\right|=\pi$. Individual susceptibilities $\chi_{s}^{b 1}$ and $\chi_{s}^{b 2}$ in eq. (3) are defined in the absence of dipole-dipole interactions and can be computed by solving standard equations for atomic density matrix elements [36]. The Rydberg populations $P^{a}$ and $P^{b}$, on the other hand, can be computed upon replacing $\Omega_{s, c} \rightarrow \Omega_{s, c} \sqrt{n_{s a} / 2}$ with $n_{s a}=N_{0}\left(4 \pi R_{b}^{3} / 3\right)$ (see footnote ${ }^{3}$ ) in the corresponding equations for SA density matrix elements. Such a scaling takes into account the fact that the signal $\{|g\rangle \leftrightarrow|e\rangle\}$ and control $(\{|m\rangle \leftrightarrow|e\rangle\}$ transitions are enhanced by the atomic number $n_{s a} / 2$ in relevant collective states of each $\mathrm{SA}$, being the atoms taken to be equally distributed between ground levels $|g\rangle$ and $|m\rangle$.

These qualitative arguments are now quantified for a realistic sample of cold ${ }^{87} \mathrm{Rb}$ atoms. While details of the procedure used to compute both individual susceptibilities 
and Rydberg populations in eq. (3) can be found in the SM for the case of monochromatic CW signal and control fields, we plot in fig. 2 the resulting cross-phase shift as a function of the common detuning $\delta$, the coupling Rabi frequency $\Omega_{C}$ (fig. 2(a)) and the dressing Rabi frequency $\Omega_{D}$ (fig. 2(b)), showing the characteristic parameter regions where $\Delta \Phi_{s}$ equals $\pi$. There we also plot the corresponding half-optical depth (see footnote ${ }^{2}$ ) showing maximal transmission $e^{-2 \kappa_{s}} \simeq 87 \%$ (fig. 2(c)) for the driving configuration $\{\Phi-\pitchfork\}$ in fig. 1(a) yet $e^{-2 \kappa_{s}} \simeq 66 \%$ (fig. $2(\mathrm{~d})$ ) for the configuration $\{\Lambda-\Lambda\}$ in fig. 1(b).

The large shifts observed in fig. 2 hinge on appreciable differences both in the i) individual susceptibilities and in the ii) Rydberg populations, as eq. (3) suggests and also confirmed in the SM through analytical and numerical computations. The former i) arises from the fact that the signal experiences different dispersions in the $\Lambda$-type single-EIT regime $(b 2)$ and the $\Lambda$-type double-EIT regime (b1) [33], whereas the latter ii) arises from the fact that Rydberg excitations, clearly occurring when the control is absent $(b 1 \rightleftharpoons b 2)$, are instead largely suppressed when the control is present $(a 1 \rightleftharpoons a 2)$. Such a quenching of the excitation probability $P^{a}$ is due to the destructive interference between the competing excitation paths $\{|g\rangle \rightarrow|e\rangle \rightarrow|r\rangle\}$ and $\{|g\rangle \rightarrow|e\rangle \rightarrow|m\rangle \rightarrow|e\rangle \rightarrow|r\rangle\}$. This competing behavior is instead absent for $P^{b}$ whereby the only excitation path is $\{|g\rangle \rightarrow|e\rangle \rightarrow|r\rangle\}$. It is to be noted that although the path $\{|g\rangle \rightarrow|e\rangle \rightarrow|m\rangle \rightarrow|e\rangle \rightarrow|r\rangle\}$ represents a high-order process, its contribution to the transition amplitude is nevertheless significant due to the enhanced SA Rabi frequencies $\left(\Omega_{s, c} \rightarrow \Omega_{s, c} \sqrt{n_{s a} / 2}\right)$ on the probe and control transitions shared by $n_{s a} / 2$ atoms.

Reaching the $\pi$ cross-phase shifts in fig. 2 thus depends on the signal and control polarizations, through a careful selection of specific dispersive EIT regimes and specific Rydberg blockade effects. This polarization-sensitive blockade mechanism, in particular, is an important and novel feature that may be easily implemented to achieve large optical cross-nonlinearities in atomic media. Such a novelty could be especially appreciated through the comparison with familiar cross-nonlinear mechanisms without Rydberg blockade. This comparison is presented in the SM, showing that the relevant $\Delta \Phi_{s}$ turns out to be orders of magnitudes smaller (as there is no appreciable differences on $\phi_{s}^{a, b}$ between the situations in which the control field is on or off) while $\kappa_{s}^{a, b}$ (indicating absorption) remain largely the same level. There we also discuss the influence of the Rydberg dephasings $\left(\gamma_{g r, m r, a r}\right)$ on absorption.

Dynamics. - In a realistic setup one should consider signal and control pulses rather than monochromatic beams. The extension is not straightforward owing to typical pulse distortion effects [37] during the propagation in a dense dispersive sample of cold Rydberg atoms [7]. So we examine in the following the intrinsically timedependent [11] cross-phase dynamics for narrow-band signal and control pulses under the same symmetric EIT
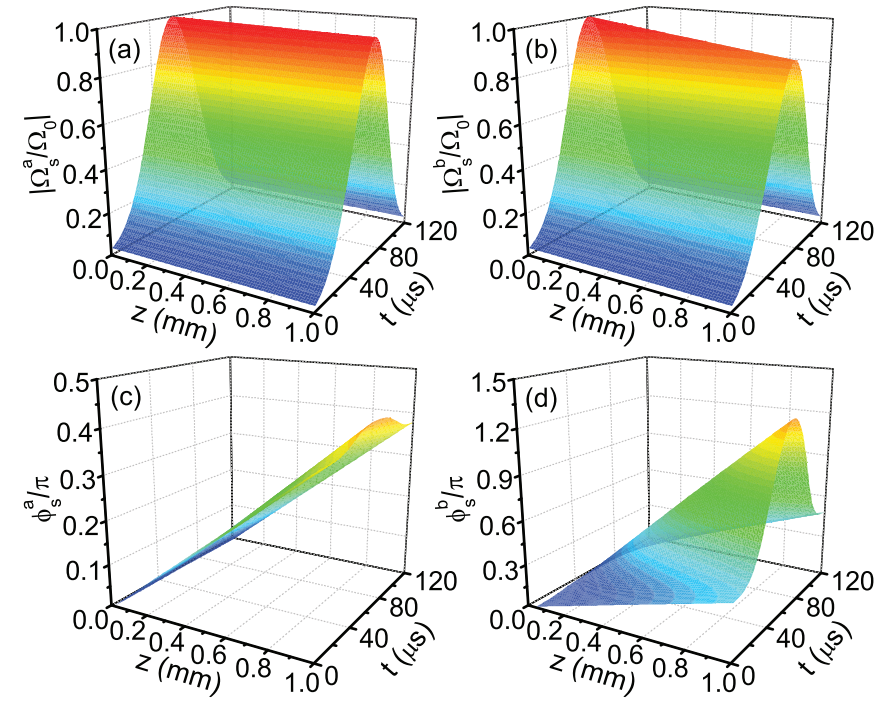

Fig. 3: (Color online) Spatial-temporal evolution of amplitudes ((a), (b)) and phases ((c), (d)) of a signal pulse in the presence $((\mathrm{a}),(\mathrm{c}))$ and absence $((\mathrm{b}),(\mathrm{d}))$ of another control pulse. Both incident pulses have the Gaussian profile $\Omega_{s, c}(t)=\Omega_{0} e^{-\left(t-t_{0}\right)^{2} / \delta t^{2}}$ with $\Omega_{0}=2 \pi \times 0.1 \mathrm{MHz}, t_{0}=60 \mu \mathrm{s}$, and $\delta t \simeq 32 \mu \mathrm{s}$. Relevant parameters are the same as in fig. 2 except $\Omega_{C}=2 \pi \times 6.0 \mathrm{MHz}, \Omega_{D}=2 \pi \times 12.0 \mathrm{MHz}$, and $\delta=2 \pi \times 80.19 \mathrm{MHz}$.

driving conditions adopted before. The signal pulse slowly varying envelope wave equations can be written as

$$
\begin{array}{r}
\frac{\partial \Omega_{s}^{a}}{\partial z}+\frac{1}{c} \frac{\partial \Omega_{s}^{a}}{\partial t}=\frac{i \pi N_{0} d_{e g}^{2}}{2 \epsilon_{0} \hbar \lambda_{s}}\left[P^{a} \sigma_{g e}^{a 2}+\left(1-P^{a}\right) \sigma_{g e}^{a 1}\right], \\
\{a 1 \rightleftharpoons a 2\},
\end{array}
$$

$$
\begin{array}{r}
\frac{\partial \Omega_{s}^{b}}{\partial z}+\frac{1}{c} \frac{\partial \Omega_{s}^{b}}{\partial t}=\frac{i \pi N_{0} d_{e g}^{2}}{2 \epsilon_{0} \hbar \lambda_{s}}\left[P^{b} \sigma_{g e}^{b 2}+\left(1-P^{b}\right) \sigma_{g e}^{b 1}\right] \\
\{b 1 \rightleftharpoons b 2\} .
\end{array}
$$

The signal pulse evolution when the control pulse is on $\{a 1 \rightleftharpoons a 2\}$ or off $\{b 1 \rightleftharpoons b 2\}$ is determined by the coupled Maxwell-Liouville equations [38], including eqs. (4), (5) and relevant dynamic equations for the atomic coherences $\sigma_{g e}^{a 1, a 2, b 1, b 2}$ and for the SA Rydberg populations $P^{a, b}$, as discussed in detail in the SM.

For a pair of identical signal and control Gaussian pulses, the signal amplitudes $\left|\Omega_{s}^{a, b}\right|$ are plotted in fig. 3(a) $\{a 1 \rightleftharpoons a 2\}$ and in fig. $3(\mathrm{~b})\{b 1 \rightleftharpoons b 2\}$. It is clear that the signal pulse experiences only slightly different losses, deformations, and time delays at the sample exit regardless of the control pulse. More interestingly, the signal phases $\phi_{s}^{a, b}=\arg \left(\Omega_{s}^{a, b}\right)$ plotted in fig. $3(\mathrm{c})\{a 1 \rightleftharpoons a 2\}$ and in fig. $3(\mathrm{~d})\{b 1 \rightleftharpoons b 2\}$ turn out to be significantly inhomogeneous or roughly homogeneous depending on whether the control pulse is on or off. It should be stressed, in particular, that $\Delta \Phi_{s}$ as inferred from fig. 3(c), (d) cannot exceed $\sim 0.85 \pi$, at variance with the value of $\pi$ predicted by fig. 2(a), (b) in the steady-state case. One main reason 
is that absorptive loss is not negligible for the signal and control pulses during propagation, which is more evident around the pulse centers.

Cross-phase shifts $\Delta \Phi_{s}$ close to $\pi$ may still be reached, e.g., through a slight change of the common detuning $\delta$ to enlarge its departure $(190 \mathrm{kHz} \rightarrow 220 \mathrm{kHz})$ from the double EIT resonance. Figure 4(a) shows the difference of individual signal phases (red dashed line), conditional to the Rydberg blockade occurrence, and the difference of SA Rydberg populations (blue dotted line), conditional to the control pulse polarization. They are so large that the corresponding cross-phase shift in fig. 4(b) displays a maximum $\sim 0.96 \pi$ (red dashed line). This maximum is nearly concomitant with the signal output peak and exhibits a $6 \%$ departure from the top for a $5 \%$ time variation around the center. It is worth stressing that the slow Rydberg decay affects further the evolution of Rydberg populations difference and thus cross-phase shift to result in a slow decay past the signal pulse. More homogeneous cross-phase shifts (blue dotted line) may be attained by using identical flat-top signal and control pulses [39]. Figure 4(c) shows that signal amplitude losses are about $8 \%$ or $22 \%$ while signal time delays are about $1.8 \mu \mathrm{s}$ or $5.8 \mu \mathrm{s}$, depending on whether the control pulse is on $\{a 1 \rightleftharpoons a 2\}$ or off $\{b 1 \rightleftharpoons b 2\}$. The SA populations in fig. $4(\mathrm{~d})$ for the $\{b 1 \rightleftharpoons b 2\}$ case follow nearly adiabatically the pulse excitation though this is less apparent at the trailing edge. One main reason is that the pulse excitation in a $X$ configuration is faster than the Rydberg decay so that repopulating the ground levels becomes a slow process. This does not happen instead for the $\{a 1 \rightleftharpoons a 2\}$ case (not shown) because the destructive quantum interference in a $\Phi$ configuration well prevents the Rydberg excitation.

It is worth noting that, for pulses containing thousands of photons ${ }^{6}$ over a beam waist of $w \simeq 12 \mu \mathrm{m}$, the results shown in figs. 3 and 4 correspond to a cross-phase shift of mrads/photon, which is an important figure of merit for tasks such as the realization of low-light-intensity crossphase modulations. Therefore, the results in fig. 4(b) definitely represent a significant achievement [32] with the sample parameters suitable to state-of-the-art magnetooptical traps [40], while the prospect of obtaining sizable cross-phase shifts even with weaker signal and control pulses down to tens of photons hinges on the availability of denser Rydberg samples, yet with appropriate dephasing rates (see $\mathrm{SM}$ for details). One main reason is that the variation of SA Rydberg populations $P^{a}$ and $P^{b}$ is determined by $n_{s a} \times \Omega_{0}^{2} / 2$ so that a smaller Rabi frequency $\Omega_{0}$ may be compensated through a larger atomic number $n_{s a}$ per blockade sphere. Our scheme could also be adapted to setups that offer a closer prospect for applications such as cold atoms loaded into hollow-core optical fibers [41]

\footnotetext{
${ }^{6}$ Phase noises for weak coherent-state incident pulses considered here can be safely neglected. Such noises may however amount to additional small dephasing rates on relevant atomic transitions when few photons are involved.
}
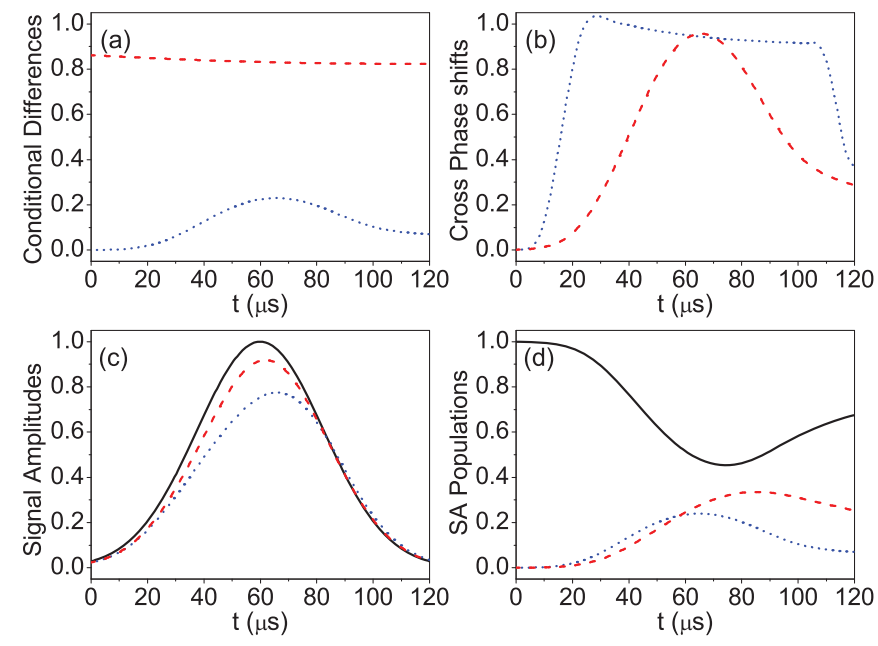

Fig. 4: (Color online) (a) Individual phases difference $0.2 \times$ $\phi_{0} \operatorname{Re}\left[\chi_{s}^{b 2}-\chi_{s}^{b 1}\right] / \pi$ (red dashed line) and SA populations difference $P^{b}-P^{a}$ (blue dotted line) at the sample exit for the Gaussian incident pulses as in fig. 3. (b) Cross-phase shifts $\Delta \Phi_{s} / \pi$ at the sample exit for the Gaussian (red dashed line) incident pulses as in fig. 3 and the flat-top (blue dotted line) incident pulses with a $\sim 90 \mu$ s duration. (c) Signal amplitudes at the sample exit in the presence (red dashed line) or absence (blue dotted line) of the control pulse, in reference to that at the sample entrance (black solid line). (d) SA populations of collective states $|g\rangle$ (black solid line), $|a\rangle$ (red dashed line), and $|r\rangle$ (blue dotted line) at the sample exit in the absence of the control pulse. Relevant parameters are the same as in fig. 3 except $\delta=2 \pi \times 80.22 \mathrm{MHz}$.

and even solid-state setups such as Rydberg excitons in cuprous oxide $[42,43]$.

Conclusions. - Specific polarization-conditional cooperative nonlinearities that occur in all-optically tunable far-off-resonance Rydberg-EIT media [26] can be harnessed to attain mrads/photon ${ }^{7}$ [44] cross-phase shifts between a signal and a control pulse. An important feature of this scheme is that a signal (control) pulse containing thousands or even less photons is already sufficient to reach $\pi$ cross-phase shifts in a regime of intrinsically small losses and group-velocity matching. We foresee this as an asset for future applications of deterministic optical gates with no need of optical cavities $[7,24,25]$, storage and retrieval [23], or post-selection procedures [14,23].

This work is supported by the National Natural Science Foundation (Nos. 11534002 and 11674049) of China, the 111 Project (No. B13013) of China, and the Cooperative program (No. PGR00960) by the Italian Ministry

\footnotetext{
${ }^{7}$ At higher densities even steeper cross-phase shifts per photon can be achieved through a fewer-photons signal (control) pulse (see SM for details). Dephasing effects, however, will start to become important with increasing densities [44].
} 
of Foreign Affairs \& International Cooperation and the National Natural Science Foundation of China. J-HW, MA, and GCLR would like to acknowledge the kind hospitality, respectively, in Pisa and in Changchun.

\section{REFERENCES}

[1] Agrawal G. P., Nonlinear Fiber Optics (Academic Press, New York) 2001.

[2] Matsuda N., Shimizu R., Mitsumori Y., Kosaka H. and Edamatsu K., Nat. Photon., 3 (2009) 95.

[3] Nielsen M. A. and Chuang I. L., Quantum Computation and Quantum Information (Cambridge University Press, Cambridge) 2000.

[4] Milburn G. J., Phys. Rev. Lett., 62 (1989) 2124.

[5] Turchette Q. A., Hood C. J., Lange W., Mabuchi H. and Kimble H. J., Phys. Rev. Lett., 75 (1995) 4710 .

[6] Reiserer A., Kalb N., Rempe G. and Ritter S., Nature (London), 508 (2014) 237.

[7] Parigi V., Bimbard E., Stanojevic J., Hilliard A. J., Nogrette F., Tualle-Brouri R., OurjoumtSev A. and Grangier P., Phys. Rev. Lett., 109 (2012) 233602.

[8] Fushman I., Englund D., Faraon A., Stoltz N., Petroff P. and Vuckovic J., Science, 320 (2008) 769.

[9] Hammerer K., Sorensen A. S. and Polzik E. S., Rev. Mod. Phys., 82 (2010) 1041.

[10] Fleischhauer M., Imamoglu A. and Marangos J. P., Rev. Mod. Phys., 77 (2005) 633.

[11] Shiau B.-W., Wu M.-C., Lin C.-C. and Chen Y.-C., Phys. Rev. Lett., 106 (2011) 193006.

[12] Li S.-J., Yang X.-D., CaO X.-M., Zhang C.-H., Xie C.-D. and Wang H., Phys. Rev. Lett., 101 (2008) 073602.

[13] Lo H.-Y., Chen Y.-C., Su P.-C., Chen H.-C., Chen J.-X., Chen Y.-C., Yu I. A. and Chen Y.-F., Phys. Rev. A, 83 (2011) 041804(R).

[14] Feizpour A., Hallaji M., Dmochowski G. and SteinBERG A. M., Nat. Phys., 11 (2015) 905.

[15] Lukin M. D., Fleischhauer M., Cote R., Duan L. M., Jaksch D., CiraC J. I. and Zoller P., Phys. Rev. Lett., 87 (2001) 037901.

[16] Balewski J. B., Krupp A. T., Gaj A., Hofferberth S., Löw R. and Pfau T., New J. Phys., 16 (2014) 063012 .

[17] Ates C., Sevincli S. and Pohl T., Phys. Rev. A, 83 (2011) 041802(R).

[18] Petrosyan D., Otterbach J. and Fleischhauer M., Phys. Rev. Lett., 107 (2011) 213601.

[19] Nielsen A. E. B. and Molmer K., Phys. Rev. A, 81 (2010) 043822.

[20] Petrosyan D. and Fleischhauer M., Phys. Rev. Lett., 100 (2008) 170501.
[21] Firstenberg O., Peyronel T., Liang Q.-Y., Gorshkov A. V., Lukin M. D. and Vuletic V., Nature (London), 502 (2013) 71.

[22] Pritchard J. D., Maxwell D., Gauguet A., WeathERILl K. J., Jones M. P. A. and Adams C. S., Phys. Rev. Lett., 105 (2010) 193603.

[23] Tiarks D., Schmidt S., Rempe G. and Durr S., Sci. Adv., 2 (2016) e160036.

[24] Hacker B., Welte S., Rempe G. and Ritter S., Nature (London), 536 (2016) 193.

[25] Beck K. M., Hosseini M., Duan Y. H. and Vuletic V., Proc. Natl. Acad. Sci. U.S.A., 113 (2016) 9740.

[26] Wu J.-H., Artoni M. and La Rocca G. C., Phys. Rev. A, 92 (2015) 063805.

[27] Friedler I., Petrosyan D., Fleischhauer M. and Kurizki G., Phys. Rev. A, 72 (2005) 043803.

[28] Gorshkov A. V., Otterbach J., Fleischhauer M., Pohl T. and Lukin M. D., Phys. Rev. Lett., 107 (2011) 133602.

[29] He B., Sharypov A. V., Sheng J.-T., Simon C. and Xiao M., Phys. Rev. Lett., 112 (2014) 133606.

[30] Paredes-Barato D. and Adams C. S., Phys. Rev. Lett., 112 (2014) 040501.

[31] Ottaviani C., Vitali D., Artoni M., Cataliotti F. and Tombesi P., Phys. Rev. Lett., 90 (2003) 197902.

[32] Li R.-B., Zhu C.-J., Deng L. and Hagley E. W., Appl. Phys. Lett., 105 (2014) 161103.

[33] Our symmetric driving may also be viewed as a symmetrically driven double-EIT configurations (see, e.g., JosHI A. and Xiao M., Phys. Rev. A, 72 (2005) 062319).

[34] Garttner M., Whitlock S., Schonleber D. W. and Evers J., Phys. Rev. A, 89 (2014) 063407.

[35] Tanasittikosol M., Pritchard J. D., Maxwell D., Gauguet A., Weatherill K. J., Potvliege R. M. and Adams C. S., J. Phys. B: At. Mol. Opt. Phys., 44 (2011) 184020.

[36] Scully M. O. and Zubairy M. S., Quantum Optics (Cambridge University Press, Cambridge) 1997.

[37] Artoni M. and Loudon R., Phys. Rev. A, 57 (1998) 622.

[38] Wu J.-H., Artoni M. and La Rocca G. C., Phys. Rev. A, 82 (2010) 013807.

[39] Venkataraman V., Saha K. and Gaeta L., Nat. Photon., 7 (2013) 138.

[40] Sparkes B. M., Bernu J., Hosseini M., Geng J., Glorieux Q., Altin P. A., Lam P. K., Robins N. P. and Buchler B. C., New J. Phys., 15 (2013) 085027.

[41] Langbecker M., Noaman M., KJaergaArd N., Benabid F. and Windpassinger P., Phys. Rev. A, 96 (2017) 041402.

[42] Kazimierczuk K., Frohlich D., Scheel S., Stolz H. and BAYER M., Nature (London), 514 (2014) 343.

[43] Artoni M., La Rocca G. C. and Bassani F., Europhys. Lett., 49 (2000) 445.

[44] Gaj A., Krupp A. T., Balewski J. B., Low R., Hofferberth S. and PfaU T., Nat. Commun., 5 (2014) 4546. 